\title{
USE BIG DATA TO INVESTIGATE PLANTAR PRESSURE DISTRIBUTION OF THE CHINESE PEOPLE
}

\author{
Su Jianhua \\ Exhibit Technology Department \\ China Science And Technology Museum \\ Beijing, China \\ acadesis@126.com
}

\begin{abstract}
Large amounts of data of plantar pressure measurement being collected by device which placed in the museum, the purpose is to observe and analyze plantar pressure distribution if within the scope of the health value. About 36000 healthy visitors are invited as the subject of this project. Participants are asked to walk as usual on a Footscan plate which is product by Rsscan International. According to authoritative statistics on the height and weight of the Chinese citizens to set device parameters. Data are processed by EXCEL. The results of the experiment revealed that most of the participants of the plantar pressure distribution are not in the normal range.
\end{abstract} Big data

Keywords-foot pressurement; gait; measurement technology;

\section{INTRODUCTION}

Aristotle (384-322 BCE) to record the human way of walking, which is has been informed of the earliest record in human walking. In 1891, Wilhelm Braune and Otto Fischer for the first time using gait analysis. Since the past century, scientists found that the relationship between the gait and plantar pressure, and continuous research and improvement of plantar pressure measurement equipment [1]. Now, through the force platform with thousands of pressure sensors can accurately measure the pressure. Footscan ${ }^{\circledR}$ system of RSscan company has been extensively used. It is widely used in fields such as medical, forensic, sport training, customized shoes, etc.

In the medical field, analysis of plantar pressure distribution can help doctors determine whether people with hallux valgus foot [2]-[4], flatfoot [5], and selects the best rehabilitation programmes for patients. Footscan system is used for foot fracture evaluation [6], and predicted training injury [7]. Now, Footscan system is also often used to provide accurate data for custom shoes to people who Athletes and the pursuit of shoes comfortable consumers [8].

Normal Plantar pressure distribution data and graphics are important in experimental frame of reference. Wu lijuan [9] was measured normal plantar pressure data of children by using Footscan system and drew a conclusion which after 7 years old (up to 11 years old) children of different gender of plantar pressure distribution did not differ. Wang mingxin [10] had collected and analyzed 100 cases of healthy adults of plantar pressure data and graphics. Footscan system itself also provides a range of health plantar pressure data and pressures graphics.

Existing related research mostly has a small number of participants, and the sample had distinct regional characteristics. Experiment environment is set as exhibits in China Science And Technology Museum. We can easily get a mass of data which is no age limit and eliminate regional of data. Engineer had developed a special software which can graph data of complex personal of real-time acquisition in order to let everyone can understand it.

In this paper, we had selected 36000 data of plantar pressure data from a large number of health invited the Chinese. These data are used to investigate the Plantar pressure distribution.

\section{METHOD}

\section{A. Subject}

The experimental device has been fixed in the exhibition hall. The visitors of visiting the museum are invited to be the participants of subject. They ranged in age from 7 to 70 . Collect the data during the summer (June/July/ August). In the season, people for the most part wear barefoot shoes (or wear thin socks), so the acquisition of plantar pressure data accurately, and during the summer vacation, the number of visitors. Obviously abnormal plantar pressure data, such as wearing shoes, leg disability, lame, history of leg injuries, had been excluded from collecting.

\section{B. Apparatus}

Equipment, Footscan gait analysis system, use in the experiment has developed by RSSCAN. The dimensions ( $\mathrm{L} \mathrm{x}$ W x H) of the device is $2096 \mathrm{~mm} \times 469 \mathrm{~mm} \times 18 \mathrm{~mm}$, and with 16384 sensors at a scanning rate of up to $500 \mathrm{~Hz}$ or 500 measurements per second. It is the largest size of the current company, the largest number of sensor and the fastest scanning The device has been firmly fixed on the stage which is composed of steel frame and three-ply board (fig.1). An antimicrobial of the rubber cushion to cover on it, and it is thin enough so that the impact on the influence of the pressure sensor can be ignored. 
Equipment does not exist independently in the exhibition (fig.2). The device is connected to a host which access local area network (LAN) with USB cable. The data of plantar pressure on LAN share with other exhibition, and it is stored by database control system of host. Speakers and monitors as well as connected to the host. The speaker used to real-time guide participant, and measurement results can be real-time display on the screen. Especially, all the equipment is in standby mode until participant use ticket of the museum (RFID card, includes unique ID Number, Age, Gender and other information) open the electronic lock.

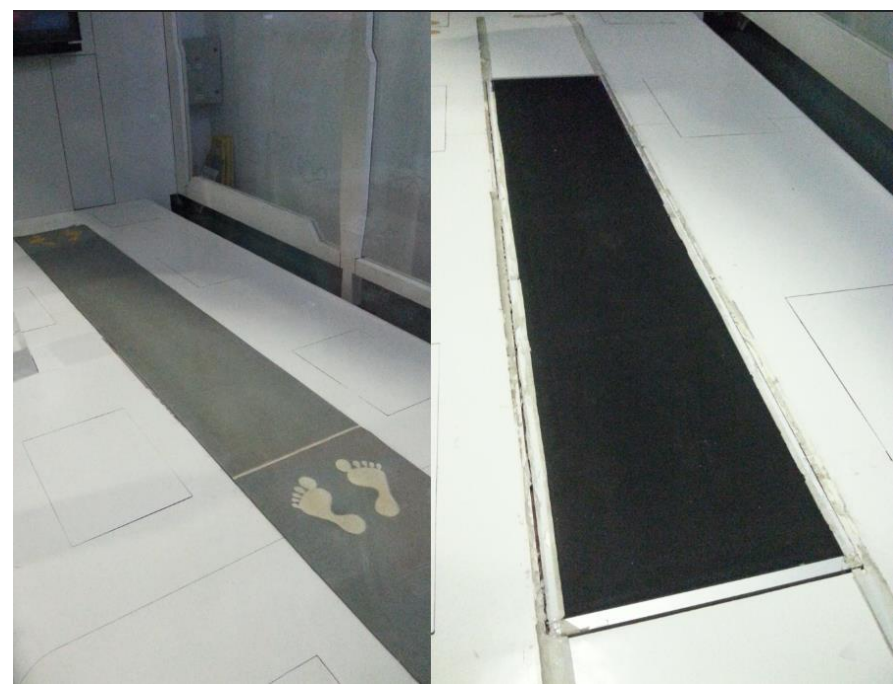

Figure.1 The high speed $2 \mathrm{~m}$ Hi-End footscan ${ }^{\circledR}$ system

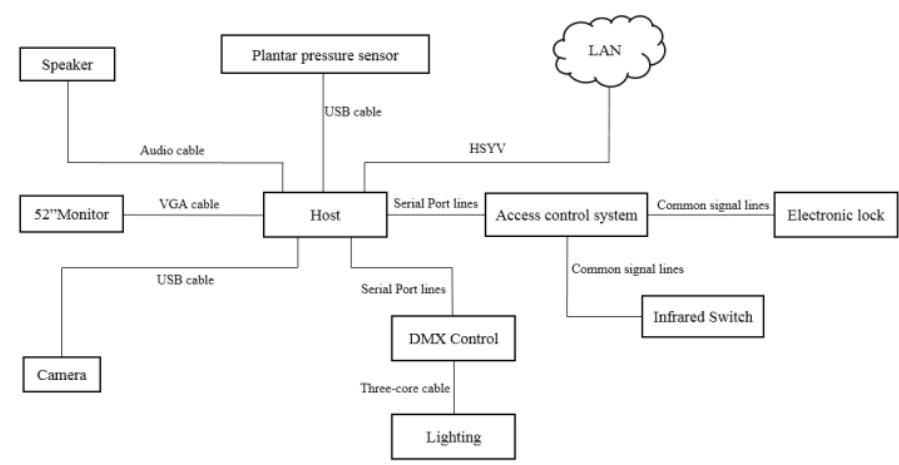

Figure.2 The exhibits electrical system diagram

\section{Experiment design}

Footscan needs to be calibrated before the experiment start: (1) Sampling time for 8 seconds, scanning speed is set to $100 \mathrm{~Hz}$; (2) According to the existing national physical data [11], we set the height as $169 \mathrm{~cm}$, the weight as $60 \mathrm{~kg}$ (Because of the huge number of participants, and the data of sampling accuracy requirement is not precision, we only input the average value). Participant standing in front of the Footscan when uses RFID cards to open the electronic lock. According to the following requirements: (1) Walk barefoot (or wear thin socks); (2) Walking relaxed as much as possible; (3) Walk straight ahead, avoiding any possible factors affecting gait, such as backpacks, twist, talk to others, etc.; (4) After leaving the force plate should continue to be at least 1 meter. Participants walked once, because the boards are long, normal gait can record four times (each foot twice).

\section{Data Recording and Analysis}

Footscan software provides a variety of plantar pressure data patterns. The analysis system of software automatically to a foot is divided into ten region(fig.3): Medial heel(HM), Lateral heel(HL), Metatarsal Heads (M1-M5), Mid foot(MF), Toe 2 to Toe 5(T2-5), Hallux(T1).

The foot force is divided into three phases: foot drop, foll over to prop up, foot lift. Normal foot walking foot contact area the largest part in the forefoot, and then the heel, Arch. Normal healthy gait, two feet of plantar pressure should be very closely (fig.4). Recording of the whole process of the change of the plantar pressure when walking through the experiment. The software records each part of plantar pressure contact pressure, contact area and contact data, calculations show it as a curve (fig.5).

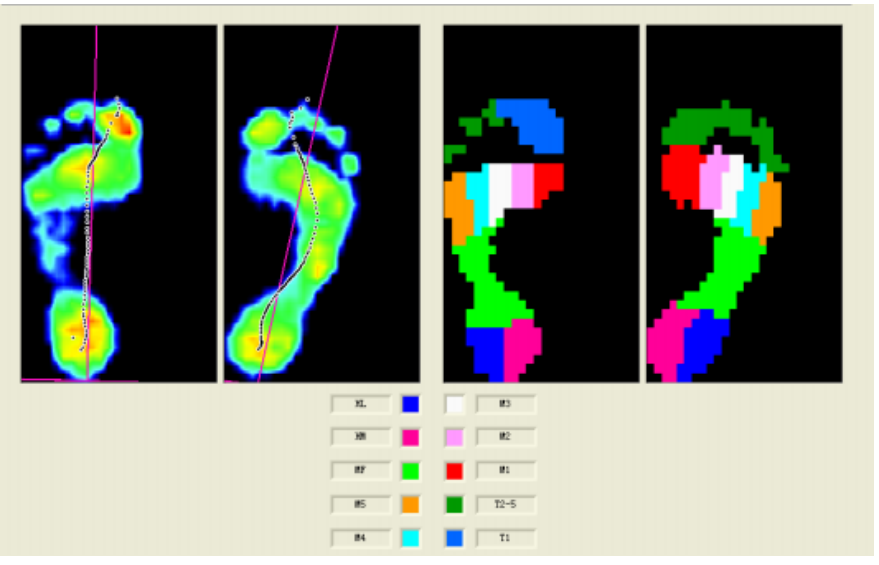

Figure.3 Plantar pressure area
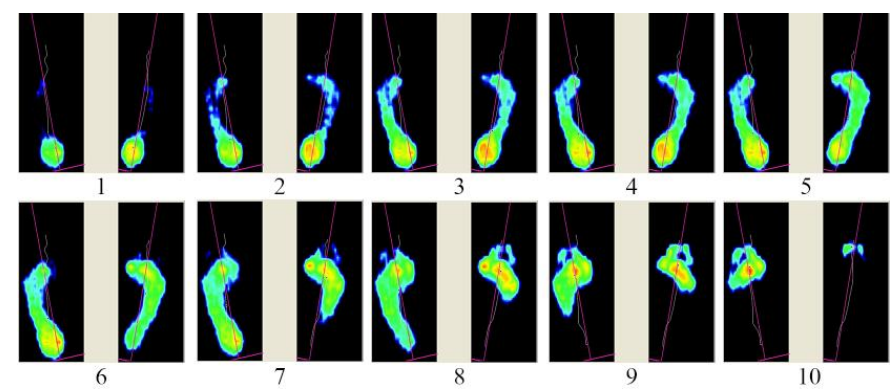

Figure.4 Normal left and right foot plantar pressure in real time images

Each zone of pelma of pressure value is simplified to a curve after statistics and calculation (Fig.6). The greater of positive values, the greater possibility of hallux valgus. Conversely, varus is more likely. If two-foot range of the pressure curve in the figure within the gray area, shows the participants of 
plantar pressure are normal, occur while walking the low risk of foot injury.
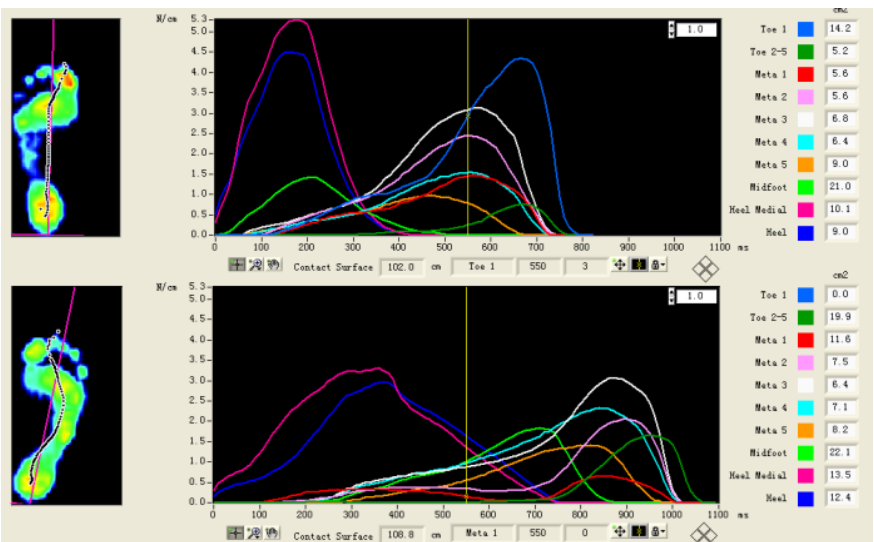

Figure.5 All regions of plantar pressure curve

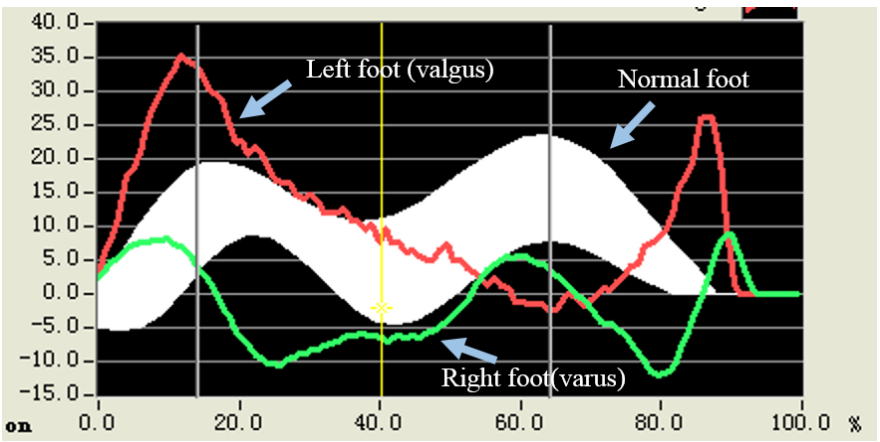

Figure.6 Plantar pressure integrated computation curves

Collected the data were grouped by gender and age. Each age group selected 3000 people. The statistical results of the data represented by histogram(Fig.7). In the histogram, black represents the number of plantar pressure normal, gray is abnormal. Obviously, the number of plantar pressure normal is far less than not normal.As the growth of the age, the number of plantar pressure abnormal increase gradually.

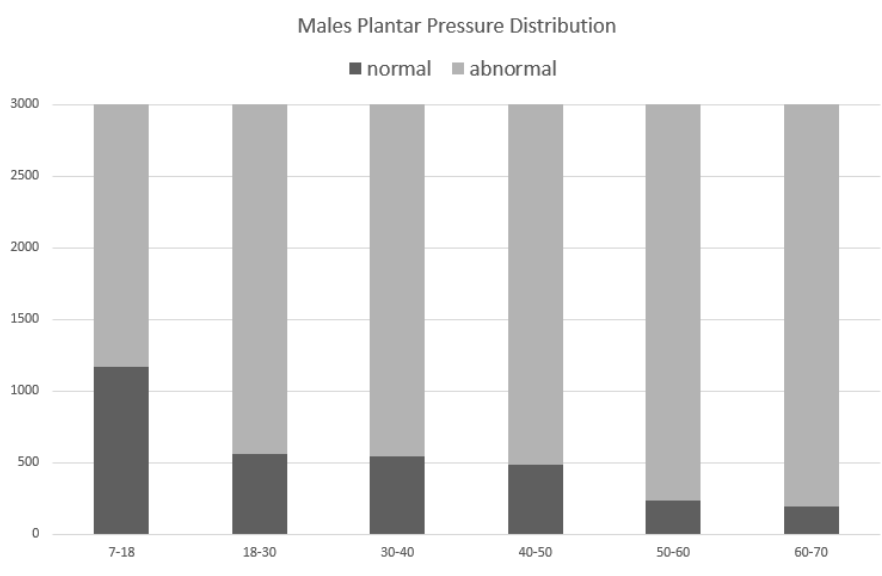

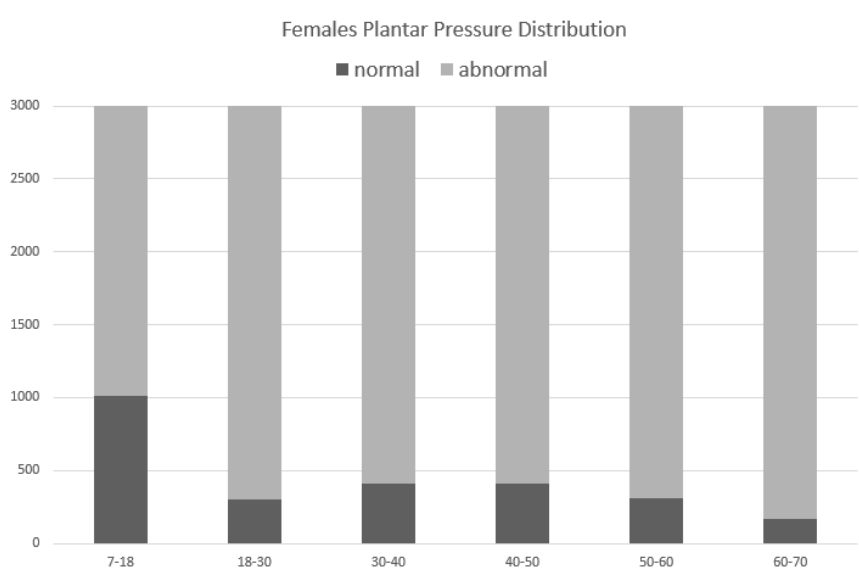

Figure.7 The statistical results of the data

\section{CONCLUSIONS}

We see from a lot of plantar pressure data, the majority of plantar pressure value is not in the normal range. The status of juveniles is better than adults. Number of plantar pressure distribution is not normal in women than men.

\section{REFERENCES}

[1] Baker R. The history of gait analysis before the advent of modern computers.[J]. Gait \& Posture, 2007, 26(3):331-342.

[2] QIE Shu-yan, ZHANG Qing-min, ZHANG Zhi-yuan, etc. Distribution changes of forefoot plantar pressure in hallux valgus [J]. JOURNAL OF MEDICAL BIOMECHANICS, 2010, 25(3):224-229.

[3] WEN Jianmin, ZHONG Honggang, JIANG Kewei, et al. Measure of the Plantar Pressure of Both Healthy Foot and Hallux Valgus Foot[J]. CHINESE JOURNAL OF ORTHOPAEDICS, 1999, 19(6).DOI:doi:10.3760/j.issn:0253-2352.1999.06.007.K. Elissa, "Title of paper if known," unpublished.

[4] Bryant A, Tinley P, Singer K. Plantar pressure distribution in normal, hallux valgus and hallux limitus feet[J]. Foot, 1999, 9(3):115-119.

[5] Du Xiao-ning, Zhao Xiao-zhe, Zhang Xin. Foot plantar pressure and gait features of flatfoot and normal foot[J]. JOURNAL OF CLINICAL REHABILITATIVE TISSUE ENGINEERING RESEARCH，2008, 12(46):9058-9061. DOI:doi:10.3321/j.issn:1673-8225.2008.46.016.

[6] CHEN Zhanfa, ZHANG Yingze, ZHENG Zhanle, et al. Quantitative evaluation of postoperative effect of calcaneal fractures using Footscan system $[\mathrm{J}]$. Chinese Journal Of Reparative And Reconstructive Surgery, 2009, 08:925-929.

[7] Franklyn A. Can RSScan footscan ${ }^{\circledR} D 3 D^{\mathrm{TM}}$ software predict injury in a military population following plantar pressure assessment? A prospective cohort study[J]. Foot, 2013, 24(1):6-10.

[8] Rout N, Zhang Y F, Khandual A, et al. 3D foot scan to custom shoe last[J]. Special Issue of International Journal of Computer and Communication Technology, 2010, 1(2-4): 14-18.

[9] WU Lijuan. The Measurements of Foot Shape Parameters and Analysis of Dynamic Plantar Pressure of Chinese Children Aged 4-11[D]. Beijing Sport University, 2011.

[10] WANG Ming-xin, YU Guang-rong, CHEN Yan-xi, et al. Analysis of the plantar pressure distr ibution of the normal Chinese adult. Tongji Hospital of TongjiUniversity, 2007.

[11] General Administration of Sport of China. Report On National Physical Fitness Surveillance(2010) [M] . People's Sports Publishing House of China, 2011 